\title{
Pregnancy-induced Bilateral Corneal Oedema
}

\author{
Datta G Pandian, Benjamin Nongrum, Renuka Srinivasan, Bhanushree Gopaliah \\ Department of Ophthalmology, Jawaharlal Institute of Postgraduate Medical Education and \\ Research, Pondicherry, India
}

\begin{abstract}
This report is of a 20-year-old woman with pregnancy-induced bilateral corneal oedema causing significant visual loss. The patient presented at 37 weeks of gestation with decreased vision and redness in both eyes. She also had pregnancy-induced hypertension, thyrotoxicosis, and rheumatic heart disease with mitral regurgitation. She was treated conservatively with tear substitutes. Following delivery, there was a dramatic decrease in corneal oedema and improvement in visual acuity. Oestrogen receptors have been demonstrated in corneal tissues, and the water-retaining property of oestrogen in tissues is well known. Pregnancyinduced increase in corneal thickness is known, but diffuse epithelial oedema has not been reported. This report highlights the importance of hormone-induced changes in the cornea during pregnancy.
\end{abstract}

Key words: Corneal edema, Pregnancy

Asian J Ophthalmol. 2011;13:19-21.

\section{Introduction}

Pregnancy induces physiological and pathological changes that can occur in both the anterior and posterior segments of the eye. The physiological changes in the cornea during pregnancy include corneal oedema with increased corneal thickness, reduced corneal sensitivity, and corneal steepening. ${ }^{1}$ The cornea is sensitive to the hormonal fluctuations that occur during the menstrual cycle, pregnancy, and menopause. ${ }^{1}$ Expression of oestrogen and progesterone receptors in the cornea has been demonstrated in rabbits, mice, and humans. ${ }^{2-5}$ Cyclical variation of corneal thickness has been reported during the menstrual cycle and pregnancy. ${ }^{1-3}$ The induced physiological changes often do not cause any visual problems except for contact lens intolerance during pregnancy. This is the first report of a pregnant woman with bilateral corneal oedema with considerable reduction in visual acuity. Clinicians must be aware of the physiological changes in the eye induced during pregnancy to avoid erroneous diagnoses and treatment.

\section{Case Report}

A 20-year-old pregnant woman presented in 2007 at 37 weeks of gestation with decreased vision and redness of both eyes for more than 1 month. She also had pregnancy-induced hypertension and thyrotoxicosis, and was receiving treatment for rheumatic heart disease with mitral regurgitation.

Correspondence: Dr Datta G Pandian, No 14, GN Palayam, Manaveli, Arumbarthapuram, Pondicherry 605006 , India.

Tel: (91 98) 9499 6285;

E-mail: dattagulnar12@rediffmail.com
Figure 1. Slit-lamp image showing diffuse corneal epithelial oedema.

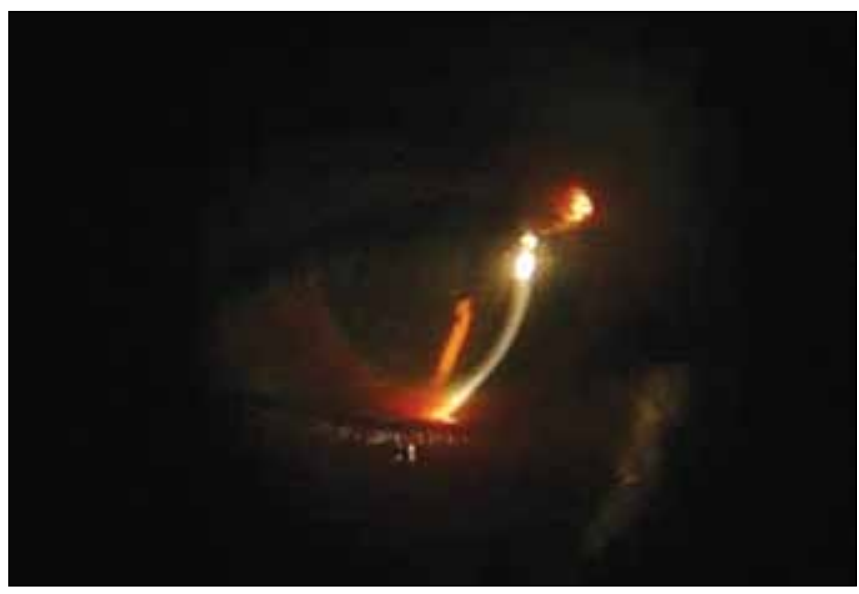

Her visual acuity was 20/200 in both eyes. Ocular examination revealed bilateral diffuse corneal epithelial and mild stromal oedema (Figure 1) with decreased corneal sensation and superficial punctate keratitis (Figure 2). She had prominent eyes (Figure 3) with lid retraction suggestive of associated thyroid eye disease. Tear function tests were normal. Intraocular pressure (IOP) by Goldmann applanation tonometry was $10 \mathrm{~mm} \mathrm{Hg}$ in both eyes. Specular reflection did not show endothelial abnormality. There was no family history of corneal disease, and she did not have a history of contact lens wear or previous episodes of redness or blurring of vision.

Her anterior chamber was clear with no cells or flare. Corneal thickness with pachymetry was $552 \mu \mathrm{m}$ and $548 \mu \mathrm{m}$ in the left and right eyes, respectively. She was treated conservatively with 
Figure 2. Slit-lamp image showing superficial punctate keratitis.

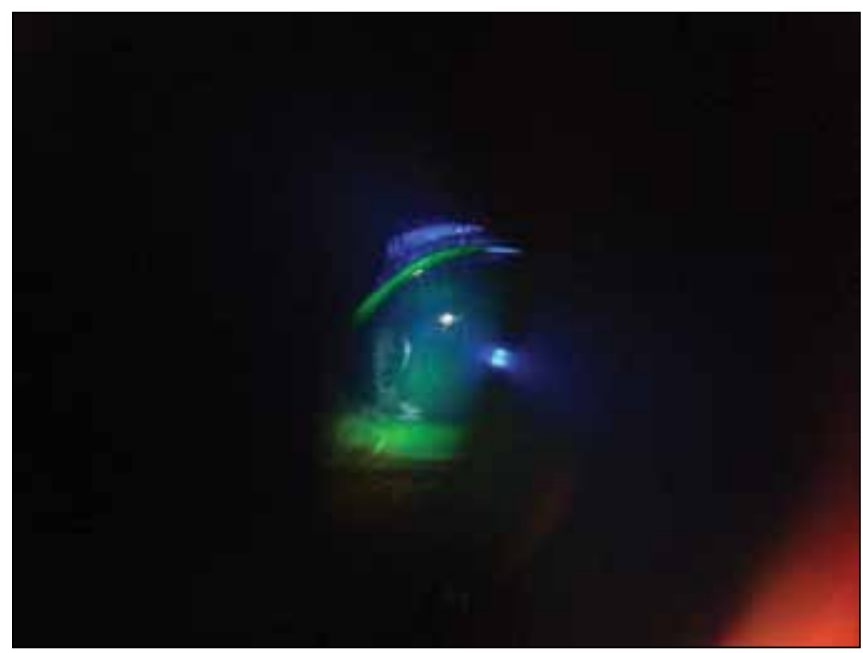

Figure 3. Prominent eye of the patient.

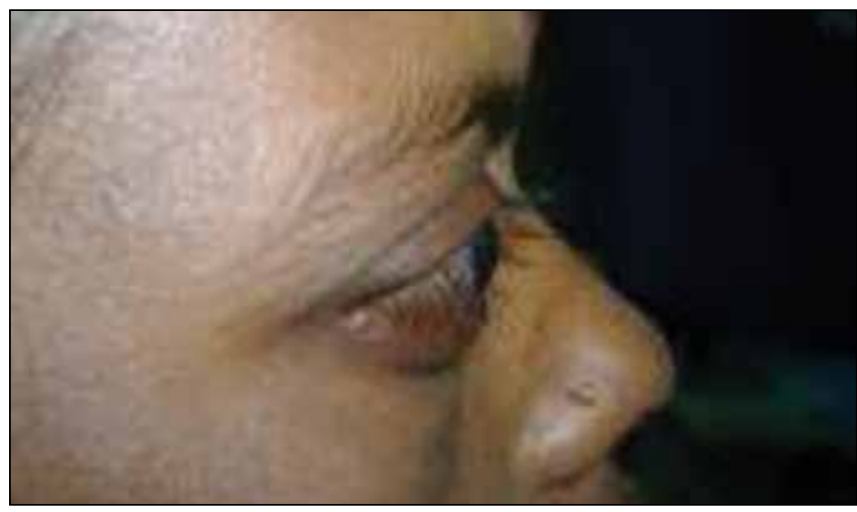

tear substitutes, and continued treatment for hypertension and thyrotoxicosis. However, there was no improvement in the corneal status until the end of the pregnancy. Ten days after delivery there was a dramatic decrease in corneal oedema with corneal thickness reduced to $468 \mu \mathrm{m}$ and $471 \mu \mathrm{m}$ in the left and right eyes, respectively. On the third day post-delivery, her vision improved in both eyes to $20 / 80$ and to $20 / 40$ after 10 days.

\section{Discussion}

The possible causes of diffuse corneal oedema with punctate keratitis in this patient include thyroid-induced changes, use or extended wear of contact lenses, herpetic keratitis, drug-induced changes, or an acute angle closure attack. The pattern of fluorescein staining was diffuse and not in the inferior or interpalpebral zone, as would be expected in exposure keratitis, especially in this patient with prominent eyes. Bell's phenomenon was adequate and there was no lagophthalmos. The patient had no history of contact lens wear. She had normal anterior chamber depth and her IOP was normal, although the measured IOP by Goldmann applanation tonometry may be erroneously low due to the presence of corneal oedema. There was no evidence of a prior acute angle closure attack, iris atrophy, or pigmentation on the cornea, and fundus examination showed no glaucomatous cupping. This suggested that the condition might be viral keratitis or physiological in origin. Since occurrence of bilateral herpetic keratitis is rare and the patient had no pain, she was treated with lubricants and closely followed up. There was no change in the staining pattern or appearance of the dendritic pattern on follow-up. The medications that she was taking (propranolol, propylthiouracil, hematinics) are not known to cause corneal oedema. Post-delivery, she was still taking these drugs and her corneal oedema cleared, suggesting that the corneal oedema might be pregnancy induced.

This report highlights a rare and reversible corneal condition occurring in pregnancy. The corneal changes were attributed to hormonal changes in pregnancy. Expression of sex steroid (oestrogen, progesterone, androgen) mRNAs has been demonstrated in various ocular tissues including the lid, lacrimal gland, bulbar conjunctiva, cornea, meibomian gland, uvea, retina, and lens. ${ }^{4}$ Steroid hormones have been detected in aqueous, tear fluid, and vitreous. Their effect is probably mediated through the sex steroid receptors in the ocular tissues. ${ }^{2}$ Studies on animals have emphasised the effect of these steroid hormones on the corneal tissue. ${ }^{1-5}$

The cornea is a collagenous tissue that is highly responsive to fluctuating hormone levels. ${ }^{2}$ The corneal stroma is rich in collagen types I and V, which are abundant in the central cornea. The hormonal influences on corneal collagen levels are greater for the central cornea than for the peripheral cornea. ${ }^{5}$ Corneal tissues are influenced more by oestrogen than progesterone, as demonstrated by the decrease in oestrogen mRNA levels in corneas of ovariohysterectomized rabbits. ${ }^{5}$

Oestrogen in tears and aqueous humour exerts its effect on the cornea through oestrogen receptors (ER), ER $\alpha$ and ER $\beta$, expressed in the corneal epithelium, stroma, and endothelium. ${ }^{2}$ Similar to its systemic effect, oestrogen in the cornea causes retention of water and sodium resulting in a cyclical increase in corneal thickness associated with ovulation and pregnancy. ${ }^{2}$ Amplification of this physiological change was seen in this patient. This increase in corneal thickness has been attributed to decreased corneal sensitivity seen with cyclical hormonal changes.

Millodot has compared corneal thickness in women during pregnancy (third trimester) and 6 to 8 weeks postpartum, and reported 30 to $50 \mu \mathrm{m}$ increases in corneal thickness during pregnancy. ${ }^{6}$ The visual acuity of these patients was not mentioned, suggesting that the increase in corneal thickness was visually insignificant. Another study has reported an average increase in central corneal thickness in gravid women of $16 \mu \mathrm{m}{ }^{7}$ 
Devastating corneal complications in compromised eyes have been reported during pregnancy, including spontaneous corneal perforation in keratoconus ${ }^{8}$ and spontaneous corneal melting in post-radial keratotomy. ${ }^{9}$ These complications are attributed to the effect of relaxin, an important hormone secreted during pregnancy, which is a positive inducer of matrix metalloproteinase that has collagenolytic properties. ${ }^{9}$

This report highlights the effect of sex hormones on corneal transparency. The spontaneous regression of the corneal haze after delivery further corroborates this possibility. There is cyclical variation in corneal thickness, curvature, and sensitivity during pregnancy and menstruation. Oestrogen plays an important role in modulating the corneal thickness, particularly in the optical zone, through receptors expressed in the corneal tissue. Although rare, the effect of these changes can be severe enough to compromise visual acuity as seen in this patient. Conservative treatment and a 'wait and watch' policy is important, as evidenced by the spontaneous regression for this patient after restoration of hormone levels following delivery.

\section{References}

1. Sunness JS. The pregnant woman's eye. Surv Ophthalmol. 1988;32: 219-38.

2. Tachibana M, Kasukabe T, Kobayashi Y, Suzuki T, Kinoshita S, Matsushima Y. Expression of estrogen receptor alpha and beta in the mouse cornea. Invest Ophthalmol Vis Sci. 2000;41:668-70.

3. Suzuki T, Kinoshita Y, Tachibana M, et al. Expression of sex steroid hormone receptors in human cornea. Curr Eye Res. 2001;22: 28-33.

4. Wickham LA, Gao J, Toda I, Rocha EM, Ono M, Sullivan DA. Identification of androgen, estrogen and progesterone receptor mRNAs in the eye. Acta Ophthalmol Scand. 2000;78:146-53.

5. Achari Y, Reno C R, Tsao H, Morck DW, Hart DA. Influence of timing (pre-puberty or skeletal maturity) of ovariohysterectomy on mRNA levels in corneal tissues of female rabbits. $J$ Mol Vis. 2008;14:443-5.

6. Millodot $M$. The influence of pregnancy on the sensitivity of the cornea. Br J Ophthalmol. 1977;61:646-9.

7. Weinreb RN, Lu A, Beeson C. Maternal corneal thickness during pregnancy. Am J Ophthalmol. 1988;105:258-60.

8. Lahoud S, Brownstein S, Laflamme MY, Poleski SA. Keratoconus with spontaneous perforation of the cornea. Can J Ophthalmol. 1987;22:230-3.

9. Arya SK, Malik A, Gupta S, Gupta H, Sood S. Spontaneous corneal melting in pregnancy: a case repport. J Med Case Reports. 2007;1: 143-7. 